\title{
Stigma and HIV concealment motivation among gay men living with HIV in Finland
}

\author{
Rusi Jaspal, PhD \\ Nottingham Trent University, UK
}

\begin{abstract}
This study explored experiences of HIV stigma among gay men in Finland and the impact of these experiences on decision-making concerning HIV status disclosure. Seventeen gay men living with HIV in Finland participated in a qualitative interview study and the data were analyzed using thematic analysis. The results focus on the following themes: (1) 'Social support impedes social stigma'; (2) 'Social support and the risk of HIV stigma', which describes how the pursuit of social support can expose some individuals to stigma; and (3) 'HIV concealment motivation as a coping strategy', focusing on the motivation to conceal one's HIV status from others to avoid stigma. It is necessary to promote awareness and understanding of HIV in Finnish society, to challenge HIV stigma and, crucially, to facilitate access to social support among those diagnosed with HIV. This is likely to have favourable implications for both psychological and public health.
\end{abstract}

\section{Keywords}

gay men; HIV; HIV stigma; identity concealment; disclosure; psychological wellbeing; Finland

\section{Introduction}

The HIV epidemic in Finland is small but has disproportionately affected gay men. Yet, there has been no published empirical research into the identities, experiences and psychological wellbeing of gay men living with HIV in Finland. Psychological wellbeing can be defined as the state of psychological equilibrium between the challenges faced in life and the social and psychological resources one possesses for dealing with these challenges effectively (Dodge et al., 2012). Social stigma associated with HIV can undermine psychological wellbeing, and the derivation of social support is a key strategy for coping with the threat of stigma. Understanding identity and experience is important both for developing strategies for enhancing the psychological wellbeing of people living with HIV and for enhancing HIV prevention efforts. Both are inextricably entwined - those with adequate levels of psychological wellbeing are more likely to engage with HIV care, to initiate antiretroviral therapy (ART) and to maintain an undetectable HIV viral load, thereby reducing the risk of onward transmission (Jaspal, 2018). This study focuses on one of the greatest impediments to psychological wellbeing and HIV prevention - social stigma - and its impact on decision-making concerning HIV status disclosure among gay men living with HIV in Finland.

\section{HIV in Finland}

Finland is a country in Northern Europe with an estimated population of 5.5 million. HIV prevalence in Finland is low, with $0.1 \%$ of the population aged between 15 and 49 thought to be living with the condition. Data show that, in 2019, 4190 people were living with HIV in Finland, of whom $1284(31 \%)$ were men infected through homosexual contact and that there were 143 new cases of HIV, of which 35 (24\%) were among men infected through homosexual contact (Finnish Institute for Health and Welfare, 2020). Gay men are thus a significant group in the HIV epidemic.

Recent data suggest that $91 \%$ of people living with HIV in Finland are aware of their serostatus, that $76 \%$ of HIV-positive individuals are on ART, and that $67 \%$ are virally suppressed (Finnish Institute for Health and Welfare, 2020). This shows that Finland has not 
yet achieved the Joint United Nations Programme on HIV/AIDS (UNAIDS) target of at least $90 \%$ on each of these three dimensions - referred to as 90-90-90. HIV care is provided free of charge to all HIV patients who can, and are encouraged to, initiate ART immediately after diagnosis. While there has been clinical research into physical health outcomes in people living with HIV in Finland (e.g. Kluger et al., 2019) and HIV epidemiology in Finland (e.g. Holmberg et al., 2019), social sciences research into the identities, experiences and psychological wellbeing of gay men living with HIV in Finland has been scarce. The role of social stigma is a conspicuous lacuna in research in this context.

\section{Research into HIV in Finland}

There is now a wealth of research into the social psychological aspects of HIV stigma and decision-making concerning HIV status disclosure (see for example Jaspal, 2018; Jaspal \& Williamson, 2017; Duffy, 2005; Earnshaw et al., 2015; Smit et al., 2012). However, there has been relatively little research in the Finnish context. Given the low HIV prevalence in Finland, HIV prevention has not generally been a public health priority in the country. However, existing research into HIV and sexual health knowledge in the Finnish population suggests that HIV knowledge is high and that respondents generally exhibit an adequate understanding of the virus and its main transmission routes (Korhonen et al., 2012; Suominen et al., 2017).

There has been just one published study of societal attitudes towards HIV in Finland, which focused on university students - it revealed a generally positive attitude toward people living with HIV (Korhonen et al., 2012). Most work has focused on attitudes toward HIV among healthcare professionals in Finland. Suominen et al.'s (2010) study of nurses' knowledge of, and attitudes towards, HIV/AIDS in Finland, Estonia and Lithuania revealed that HIV knowledge was highest and attitudes most favourable among Finnish nurses. Moreover, Välimäki et al. (2010) found a general willingness among future nurses and midwives in Finland to care for HIV patients, indicating low levels of HIV stigma in the healthcare profession. Research elsewhere has shown that HIV stigma can undermine psychological wellbeing and lead to self-isolation among patients (Varni et al., 2012). However, there has been no empirical insight into HIV stigma in Finland and, thus, it is unclear whether and, if so, to what extent this plays a role in the experiences, identities and wellbeing of gay men living with HIV in Finland.

In a study of 440 people living with HIV in Finland, Nobre et al. (2018) found that patients with severe self-stigma reported low overall quality of life and health-related quality of life. Their research showed that cohabitation with an intimate partner and HIV status disclosure were negatively associated with self-stigma, suggesting that these may be protective factors. In another study, Nobre et al. (2016) examined the social networks of nine older adults living with HIV in Finland and found that the acquisition of social support, particularly from friends and family, was the most significant coping strategy. There were concerns about ageing with HIV and the adverse impact that this may have on the availability of social support in the future.

These studies are consistent with research that shows the benefits of social support in assuaging HIV-related stressors (e.g. Earnshaw et al., 2015). Yet, in the absence of empirical research in Finland, there is limited knowledge about decision-making concerning HIV status disclosure, which is a precursor of the acquisition of social support. Moreover, the availability, nature and perceptions of social support among gay men living with HIV in Finland are unclear, and the ways in which possible experiences of social stigma may affect social support have not been ascertained. Accordingly, this study focuses specifically on the role of social stigma in the lives of gay men living with HIV and the impact that this can have for their identity, experience and psychological wellbeing. There has been very limited research into HIV stigma 
in Finnish society and, thus, this significant theme is explored, in an exploratory manner, through the qualitative accounts of gay men living with HIV in Finland.

\section{Method \\ Participant recruitment and data generation}

This study reports the findings of a semi-structured individual interview study with 17 gay men living with HIV in Finland. The interviews were conducted between September 2017 and February 2018. The participants all self-identified as male and as gay, and were aged between 36 and $72(M=50.2, S D=11.2)$. Fifteen participants identified as ethnically Finnish, one as Latin American and one as being of mixed heritage. All but one of the interviewees were born and raised in Finland.

Individuals confirmed that they had been living with diagnosed HIV for between 1.5 and 26 years $(M=14.4, S D=9.1)$. All of them were engaged in HIV care, on ART and virally suppressed. Sixteen lived in Helsinki and one in Tampere. In terms of relationship status, ten individuals reported being in a relationship; five as single; and two as 'dating'. Participants were recruited at either the Finnish HIV Foundation/ Hivpoint or Positiiviset ry HivFinland, which are Helsinki-based charities that provide HIV prevention and peer support/counselling services, respectively, for people in Finland. Sixteen interviews were conducted in person by a research assistant at the aforementioned charities and one was conducted over Skype by the author.

Interviews were guided by a semi-structured interview schedule which explored the following topics: self-description, self-categorization, identity, the experience of HIV diagnosis, HIV knowledge, the post-diagnosis period, HIV disclosure, stigma, coping, and engagement with HIV care and treatment. The interviews lasted between 50 and 90 minutes and were digitally recorded and transcribed verbatim.

\section{Analytic approach and procedure}

The data were analyzed using qualitative thematic analysis. Thematic analysis is a flexible qualitative analytic approach which allows the analyst to examine both experiential and discursive aspects of participants' accounts. It allows the analyst to generate 'themes' that shed light on patterns of meaning in the dataset (Jaspal, 2020). This is especially useful in the context of identities which are complex, dynamic and both internally represented and externally performed (Warner \& Shields, 2013).

Initial analysis was carried out by the author who followed the nine principles of thematic analysis outlined by Jaspal (2020). During the preliminary stages of analysis, impressions and interpretations on the data were noted. These emerging codes focused on participants' descriptions and interpretations of their identities and experiences, particular forms of language, and apparent contradictions and patterns in each interview. The codes were further developed with the introduction of psychological constructs (e.g. self-esteem, identity) as interpretative lenses. The emerging codes were discussed with members of the analyst's research group and any potentially idiosyncratic interpretations of the data were curbed. This also helped ensure that the codes and emerging themes could be verified and validated by other researchers in the group. Subsequently, these more developed codes were collated into potential themes with subsequent higher-level interpretative work consistent with the initial research question focusing on the impact of HIV stigma on decision-making concerning HIV status disclosure. Specific data extracts are illustrative of the themes. Participants' names have been replaced by pseudonyms. 


\section{Results}

In this section, the following three themes are described: (1) Social stigma impedes social support; (2); Social support and the risk of HIV stigma; and (3) HIV concealment motivation as a coping strategy.

\section{Social stigma impedes social support}

Most participants reported experiences of social stigma in relation to HIV, and attributed HIV stigma to the 'silence' and 'shame' surrounding HIV in Finland:

Whereas in some greater countries this particular topic [HIV] is discussed a lot more openly, here in Finland, we just seem to have this kind of, well, shame culture really.... Obviously that's something which makes discussing things like these openly that much harder. (Tuomas)

I'm just finding silence about HIV as nobody talks about this or their love life even. It's just silence and this makes us all think it's wrong, like a prohibited topic in their society. (Miguel)

Some interviewees described a cultural norm in Finnish society which reportedly impeded open discussion of intimate aspects of one's life, including one's HIV status. Tuomas referred to this as a 'shame culture'. Interviewees construed the 'silencing' of HIV as stigmatizing and noted that this led to the belief that HIV was a 'prohibited topic' in society. This in turn can accentuate HIV stigma (Duffy, 2005).

Participants described experiences of HIV stigma in various contexts, including in healthcare settings:

I remember coming...entering the room [in the clinic], everything was covered in...it looked more an operation room and I remember also the dentist, him-or herself...I wasn't sure if the person in front of me was a man or a female...because the person was all covered. Looked more like a butcher. (Elias)

I felt pretty kind of down and no libido and nothing like this, and she [the doctor] was something like "Of course you don't have to have a libido. It's not a problem if you don't have libido!" and I was like "Well if you don't have a libido and you don't find it a problem, fine, but I don't have a libido, and I feel pretty tired, it's a problem for me!'. And I really sensed that, as a gay HIV-positive man, for her it was wonderful that I didn't have a libido. (Jesse)

Some individuals construed the behavior of their healthcare providers as stigmatizing. Elias believed that his dentist had worn extra protective clothing due to his HIV positive status, which he found inappropriate. In his interview, Elias proceeded to describe how this experience reinforced his feelings of self-stigma in relation to this HIV status. Jesse described the psychological distress associated with his loss of sex drive, which he attributed to his initiation of ART. Jesse construed the doctor's response as indication that she believed that it would be preferable for him, as an HIV-positive gay man, not to have sexual relations with others. In short, he understood the doctor's conduct to be symptomatic of HIV stigma.

Participants also described stigmatizing responses from family members to whom they disclosed their positive serostatus. For Aleksi, his parents' active denial of his HIV status reflected HIV stigma: 
I said "Hello, I have something to tell, I have HIV.". No response and they put the TV on louder so they kind of ignored it, they didn't want, then I thought maybe they don't understand what HIV is, so I shouted 'I HAVE AIDS!' And then they put the TV on louder... then, they shut it off and my mother looked, and she said "I have to do some laundry now"... My father was just sitting, my image was that my mother started to cry... Then on that day we didn't talk about that at all. My father asked "could I have got it because we'd been in the sauna together, and you have been using my [mimicking shaving]?' (Aleksi)

Aleksi and other interviewees expressed their need for interpersonal support but noted that this was unavailable in the immediate aftermath of their diagnosis. Indeed, the family is commonly an anticipated source of social support in the aftermath of psychological adversity (Davey al., 2009). Yet, Aleksi did not feel supported by his parents who reportedly preferred to avoid knowledge of his HIV status. His mother's response, initially characterized by denial, later developed into distress, while his father's response was to check whether he himself was in danger of infection. Social support consists of reassurance from significant others that one will be able to cope effectively with the adverse situation but the parental response to Aleksi's disclosure was characterized by silence, despair and fear. Such a response might reinforce the death anxiety and fear of onward transmission that commonly accompanies an HIV diagnosis (Miller et al., 2012; Peyre et al., 2019).

In addition to denial, indifference was a common behavioral response from significant others, which served to reinforce HIV stigma. Juri reported disclosing his HIV status to a colleague from whom he sought support:

I told one of my gay friends and a few of my colleagues, who took it well except for one who reacted like "Why are you telling me this?". She didn't understand why I told her this thing. Because it's said that in the states of a crisis you desire to talk, in a way, and I talked maybe to too many people in a way. (Juri)

Juri himself acknowledged that self-disclosure could provide some respite from psychological adversity. When he disclosed his HIV status to his work colleague, she reportedly questioned the logic of sharing this sensitive information and could not understand the psychological distress that Juri might be experiencing as a result of his diagnosis. This may stem from decreased awareness and understanding of HIV in Finland, on the one hand, but also to the 'shame culture' in Finnish society, to which Tuomas made reference in his account. Juri had sought interpersonal support but encountered indifference, which reduced the perceived availability of social support. For Juri and others who described negative responses to their disclosure experiences, such experiences reinforced the perception of HIV stigma. Moreover, they indicated implicitly that one's HIV status ought not to be shared with others and that it was futile to expect social support.

\section{Social support and the risk of HIV stigma}

There is evidence of psychological distress associated with HIV status disclosure to sexual partners, which arises from the stigma, rejection and hostility that people living with HIV can encounter (Smit et al., 2012). Interviewees unanimously described HIV stigma upon disclosure of their serostatus:

In my experience, I haven't gotten a positive reaction when I tell it. It's always been a little bit like, like a difficult situation, because my dates are outsiders and maybe they are not so.. They haven't had to think about it so much. (Juri) 
For me, telling is a nightmare because of my fear of what will happen next. It's like I am damaged or dirty. I never had a good response, maybe a mediocre response but never good. The best scenario they say 'OK, thank you but no thank you' (Miguel)

Juri and Miguel reported that their experiences of HIV disclosure had been difficult in view of stigmatizing responses from sexual partners. Juri described these experiences as 'difficult', which he attributed to the lack of awareness and discussion of HIV among the gay men whom he frequented. Conversely, Miguel who did socialize on the gay scene reported frequent exposure to HIV stigma from other gay men. He described HIV disclosure as 'a nightmare' due to his fear concerning its consequences - later in his interview, he described hostility from sexual partners who accused him of deliberately trying to transmit HIV to others. On a psychological level, even subtle rejection reinforced the self-stigma that Miguel and other interviewees reported, namely that one is 'damaged or dirty' as a result of one's positive serostatus. This was clearly aversive for self-esteem.

In view of the stigma experienced in face-to-face contexts, many individuals prefer to reveal their HIV status in online forums where they have greater control to manage their status disclosure and the nature of their interactions in contexts of disclosure. Interviewees described HIV stigma online and its psychological effect on them:

I would also add to my own profile information about being HIV-positive...I started to notice other people sending me some death threats... as a result of that, I decided to leave the whole [website] for good and that was basically it. But still you know that particular experience started to haunt me afterwards, that there were, actually were, people out there who reacted like that. (Mirko)

When you are blocked because of HIV, it is a very painful thing for a person who is positive. HIV is difficult because you could die from this. The stigma is also very difficult because you can die alone... I deleted the app. (Miguel)

The Internet constitutes an important forum for gay men in search of social support in relation to their sexual identity and HIV status (Peterson, 2009). Yet, both Mirko's and Miguel's experiences of stigma online clearly undermined their perception of the Internet as a beacon of social support. Mirko reported sharing his HIV status on a gay dating website, in response to which he received death threats from other website users. Although these could be isolated incidents, they clearly caused Mirko psychological distress, creating an anticipation of stigma upon HIV status disclosure - indeed, he noted that the experience 'haunted' him because it constituted evidence of HIV stigma among gay men, which he had not anticipated. Crucially, the experience resulted in his departure from the website, which meant that this no longer could function as a source of social support for him.

Similarly, Miguel, who used a geospatial gay mobile application to engage with others in the absence of face-to-face social support, resorted to deleting the application due to the adverse psychological impact of his stigma experiences. In contrast to Mirko's experience of overt hostility from other users, Miguel reported rejection on the application when he revealed his HIV status to other users. Experiences of being 'blocked' by other users on the application led him to believe that he would continually be rejected and ultimately 'die alone', which in turn led him to delete the application. Like Mirko, Miguel was no longer able to rely on online forums for social support. 
Interviewees described HIV stigma in their intimate relationships, which culminated in relationship dissolution, thereby curtailing a significant source of social support (e.g. Henry et al., 2015):

That relationship ended and of course he told me that this [HIV] was the reason, which wasn't so nice. (Juri)

I met a lot of guys over here and a lot of them were afraid of me...I have that stigma that when I try to have sex with someone, even if I say I'll use a condom and they're still afraid of me... It's very frustrating and I feel that, yeah, it is a kind of stigma to me. (Toni)

Individuals who reported relationship breakdown due to their HIV diagnosis experienced psychological distress. Relationship breakdown was often attributed to HIV stigma from a serodiscordant partner, which in turn led individuals to internalize this stigma and to construe it as an impediment to their intimate relationships. Furthermore, several interviewees noted that, because HIV is feared in the gay community, they too were feared as people living with the condition. Toni described this fear - both of his HIV infection and of him as an HIVpositive person - as 'a kind of stigma'. He noted that even the offer to use a condom was insufficient to assuage the concerns of sexual partners who nevertheless were 'still afraid'. Stigma of this kind caused distress. Some interviewees responded to this pervasive stigma by disengaging from others, especially potential intimate partners:

I was living in Finland back then but the stigma, the fear of, of other guys within the Helsinki gay community to know about my status, I was too afraid of getting discriminated so I opted to run away...And I decided to move to London ...I didn't even dare to come to Positiviviset in 2004...First of all, I didn't know how to react if I met somebody I know, and I would say maybe the biggest fear was that if somebody recognizes me, he or she doesn't keep quiet, word gets out...so that was my main fear... There was a rumor within the gay community in Turku that "be careful with that guy" and "that guy has HIV". (Elias)

Stigma and rejection due to HIV status led some individuals to experience decreased selfesteem. Elias decided to shield himself from stigma by 'running away' - he left the country and, when he returned, he refused to engage with HIV support services for fear of being identified and having his HIV status involuntarily disclosed to others. Elias was fearful of rumors concerning his HIV status - especially in Turku, his city of origin. While other interviewees described their disengagement from online forums in order to avoid exposure to HIV stigma, Elias decided to disengage from the gay scene in Helsinki, HIV support services and ultimately his country. The strategy of escapism was designed to limit exposure to HIV stigma and to protect his self-esteem. Yet, in the process of doing so, he abrogated any potential source of social support and initiated a process of self-isolation.

\section{HIV concealment motivation as a coping strategy}

In view of HIV stigma, most interviewees expressed what can be described as an 'HIV concealment motivation', that is, they expressed a preference to conceal their HIV status from significant others, which was motivated by the fear of stigma, rejection and hostility. They came to fear HIV disclosure: 
The illness itself, fortunately nowadays it's fairly easy to live with it, but what's very difficult is this stigma, the fear of getting discriminated, and that applies also to me, like...I was interviewed [for a newspaper] as an employee of the association, not...I didn't want to be open about myself living with HIV because...I don't want to risk getting discriminated.' (Elias)

Here in Finland, revealing that same type of information about yourself [HIV status] could potentially be causing terrible kinds of emotions within some people. (Tuomas)

Interviewer: Yeah. Is this [HIV disclosure] something which causes you to think a lot? Pessi: Well yeah, a lot actually. A lot. Aside from those people whom I already mentioned, I really couldn't see myself revealing my own diagnosis further to anyone else.

Elias and others contrasted the physical experience of living with HIV with the psychological experience of doing so, in order to exhibit the insidious psychological effects of HIV stigma. Tuomas hesitated about disclosing his status to others because of the potential emotional impact on others. Elias had clearly experienced distress in response to stigma and therefore limited the extent of his HIV disclosure. In a newspaper interview, he presented himself 'as an employee' of an HIV charity, rather than as a person living with HIV, in order to avoid stigma. Pessi constantly ruminated about HIV disclosure given that he had not disclosed his status to significant others. This posed a dilemma for Pessi who believed that he should do so.

Some respondents acknowledged the distress caused by rejection by potential sexual partners, but nevertheless believed that it was necessary to disclose:

I try to find somebody else but it does limit your, because then the next one has to be told again...it just hurts a little bit. (Toni)

Mostly I just remember thinking to myself that 'OK, I'm going to have to tell my partner about it.' You know, that was my very first thought probably and obviously also the hardest part of it all, namely because I wasn't able to inform him right away. (Mikael)

Although I did not at first have the courage to actually tell him [his partner] that [about his diagnosis]...it probably took me around three years or something like that to, you know, actually confess to him my own diagnosis. Obviously he was a bit upset about that, enquiring as to why I wasn't able to let him know about my own condition sooner than so and I also felt embarrassed for not having informed him earlier. (Mikko)

Like Toni, several interviewees believed that their HIV diagnosis had curtailed their intimate lives and reduced the likelihood of finding intimate partners. HIV disclosure was construed as a barrier to developing intimate relationships. Mikael and Mikko delayed informing their respective partners about their HIV status due to fear of rejection - in fact, Mikko finally disclosed his status three years after his diagnosis. While construed as a strategy for limiting the risk of rejection, delays in HIV status disclosure did induce negative emotions. This posed a dilemma for individuals who, on the whole, believed that it was important to disclose their HIV status while attempting to avoid the psychological distress that disclosure often induced. Some negotiated the scope of their disclosure:

I would nevertheless tell about my own HIV status. Or at least I would, you know, strive to do so...I don't want to end up in that same situation as before, namely being in a 
love relationship in which, I'm not capable of telling my own partner about it. Although, if we're talking about just some, you know, random sex experience with someone, a one-night stand of some kind, then maybe I wouldn't bother. (Mirko)

Most HIV-positive people never tell about their status in Finland, so, yeah, there is a stigma. Yeah, and one thing is also that if you tell about your status, you can be blackmailed. (Toni)

Some participants did endeavor to disclose their HIV status to sexual partners but acknowledged that, under some circumstances, they might refrain from doing so. Mirko reasoned that, while he would disclose his HIV status in a potential romantic relationship, he might not disclose to a casual sexual partner. Thus, type of partner was an important determinant of status disclosure. Toni further rationalized his decision to limit his HIV disclosure by noting that disclosure in Finland was rare, thereby constructing non-disclosure as a pervasive norm, and that HIV status disclosure carried the risk of blackmail. Nondisclosure of one's HIV status was therefore normalized as a self-protection strategy in the face of both social stigma and potential blackmail.

In view of the social stigma and ensuing psychological distress, some interviewees simply decided against HIV disclosure:

Well it [HIV stigma] feels terrible... I remember thinking to myself that I would never ever tell anyone about my HIV again. And I started to reflect on the possibility that it would never actually infect anyone...so I basically reasoned that, like I already said, that I would never tell anyone about it again. Because if I did, I would basically be risking the very possibility of ever actually meeting anyone again and ending up alone. But then again, I always kept coming back to that kind of a feeling of moral responsibility, you know, not keeping my own partners in the dark about it, and so eventually I just stopped reaching out for new people. (Jaako)

I really do not have the energy to go out on dates with people, wait for the right moment to tell about my diagnosis, and then all of a sudden witness my date running away with a shrieking voice, I mean, that kind of a humiliation is really not something which I wish to be exposed to. (Mikko)

Like most other interviewees, Jaako and Mikko described the adverse psychological impact of HIV stigma. Jaako exemplified this with an anecdote of his romantic involvement with somebody who ultimately rejected him due to his HIV status. This distressing experience led to his decision to conceal his HIV status from potential sexual partners in the future, which he rationalized by noting that people with a suppressed HIV viral load are uninfectious. ${ }^{1}$ Jaako and others expressed a fear of stigma, rejection and loneliness due to their HIV status, and Mikko reported no longer feeling emotionally empowered to disclose his HIV status to others. The decision to conceal one's HIV status was a direct consequence of pervasive social stigma.

In view of the common HIV concealment motivation, there was dismay among participants at some of the physical 'markers' of HIV infection, which were believed to increase their identifiability as HIV-positive individuals:

In San Diego you could see when we were HIV-positive because of lipodystrophy on the face. Here of course I can always think that they think I'm so old. That's why when I came here, I thought 'Shit, I have to come here with this HIV face'. (Toni) 
All of those physical marks [of Kaposi's sarcoma] on my own body...they were all really like, hard for me to accept, namely you know, due to the fact that those marks were actually there to stay for good...I have also spent countless hours online trying to search for relevant information regarding different types of lasers [to remove the scarring]...going through this whole process has actually been the most traumatic experience in my whole life so far... I started covering up my body with tattoos and I have actually been really happy with the outcome of that. (Tuomas)

As a recipient of first-generation ART in San Diego in the 1990s, Toni suffered from lipodystrophy, a common side-effect of older ART which entails visible abnormal fat distribution. Toni was concerned about the prospect of returning to Finland 'with this HIV face' because he believed that this might compromise his ability to conceal his HIV status from others. Indeed, he derived some consolation from his ability to attribute his appearance publicly to the ageing process, rather than to HIV. Similarly, as a long-term AIDS survivor, Tuomas had had Kaposi's sarcoma which causes purple masses on the skin. The experience of Kaposi's sarcoma was distressing for Tuomas due to the permanence of the scarring, which he regarded as a physical marker of HIV.

Both the experience of living with physical scarring and his futile attempts to have them medically removed were described as 'the most traumatic experience in my whole life'. In order to conceal these physical markers of HIV and, by extension, his HIV status from others, Tuomas resorted to getting multiple tattoos which provided some respite from the psychological distress of being visibly HIV-positive. Thus, there was an attempt to conceal not only one's HIV status but also the physical 'markers' of infection that might increase one's identifiability as somebody with HIV.

\section{Discussion}

In the pursuit of social support from significant others, such as friends, colleagues and family members, gay men living with HIV in Finland may encounter social stigma in relation to their HIV status. Moreover, in view of Finnish legislation that requires people living with HIV to disclose their HIV status to their sexual partners (Hivpoint, 2015), most interviewees perceived a legal and moral responsibility to do so. HIV disclosure did indeed expose interviewees to significant HIV stigma, and many did not feel that they possessed the social and psychological resources to cope effectively with this stigma and thus experienced decreased psychological wellbeing (Dodge et al., 2012).

Consequently, some people in this position did develop an HIV concealment motivation, which functioned as a strategy for coping with actual and anticipated stigma and the psychological distress that this could entail. Mirroring the concealment motivation in relation to sexual orientation (Jackson \& Mohr, 2016), HIV concealment motivation refers to a preference to conceal one's HIV status from significant others as a self-protection strategy against stigma, rejection and hostility. Much empirical research shows that the acquisition of social support is an effective and sustainable strategy for coping with psychological adversity, such as an HIV diagnosis (e.g. Earnshaw et al., 2015; Nobre et al., 2016). In view of the HIV concealment motivation, gay men living with HIV in Finland may have decreased access to social support and a limited capacity to cope.

The focus on gay men in the HIV epidemic in Finland has meant that there has been limited discussion of HIV in mainstream Finnish society, and that there may be decreased awareness and understanding of the disease in the general population (cf. Korhonen et al., 2012; Suominen et al., 2017). Interviewees also noted that Finnish cultural norms are not generally conducive to open discussion about one's private life, which in turn can impede the disclosure of HIV (e.g. Nishimura et al., 2008). It is noteworthy that, when issues are not 
discussed openly, the social representations that surround these topics can remain static and unchallenged, leading to their entrenchment in societal thinking (Duffy, 2005). This undoubtedly provides the foundations for HIV stigma.

HIV stigma was a consistent theme in participants' accounts of living with HIV in Finland, which is in contrast to a recent study of university students in Finland suggesting that attitudes are largely positive (Korhonen et al., 2012). From the perspective of the gay men living with HIV, HIV stigma is pervasive in Finnish society - theirs is an important perspective as HIV stigma has a significant impact on their experience of living with HIV and on their psychological wellbeing. There is already some evidence of self-stigma among people living with HIV in Finland (Nobre et al., 2018). The present study provides additional evidence concerning the potential antecedents of self-stigma, namely the pervasive social stigma that they encounter - especially in the pursuit of social support. HIV stigma could be attributed to the 'invisibility' of HIV as a public health topic and the silence that surrounds the condition. People living with HIV are similarly invisible and, thus, there is limited awareness and understanding of their lived experience. Silence and invisibility can fuel stigma.

Participants reported HIV stigma in interactions with family members, friends, work colleagues, and healthcare professionals. This finding is in contrast to research into attitudes towards HIV among healthcare professionals in Finland, which generally reveals favorable attitudes towards patients and a willingness to treat them (Suominen et al., 2010; Välimäki et al., 2010). The present study indicates that gay men living with HIV in Finland may perceive or anticipate HIV stigma even from healthcare professionals. In some cases, individuals may misattribute potentially innocuous events and responses from others to HIV stigma, which, due to prior experiences, they are perhaps primed to detect. However, their perspective is important because, overall, perceived stigma in healthcare may compromise the quality of, and satisfaction with, healthcare and lead to disengagement, which in turn is associated with poor individual and public health outcomes (Daramilas \& Jaspal, 2017).

HIV stigma from intimate partners was especially aversive for psychological wellbeing. This led interviewees to internalize stigma, to fear rejection and loneliness and, in some cases, to fear for their safety due to hostility from others. Many disengaged from the gay scene or from online dating forums because they anticipated HIV stigma in these contexts. Therefore, they also disconnected themselves from potential sources of social support. This amounts to a form of self-isolation, which may present short-term benefits although its longterm efficacy as a coping strategy is limited. HIV stigma presented a dilemma - on the one hand, individuals felt obliged to disclose their HIV status but, on the other hand, HIV stigma caused psychological distress, that is, most participants lacked the social and psychological resources to withstand the negative effects of stigma.

In view of actual and anticipated HIV stigma and the distress that this can cause, gay men living with HIV in Finland may exhibit an HIV concealment motivation, precluding status disclosure to significant others and to intimate partners. This self-protection strategy may shield the individual from social stigma, and thus from threats to self-esteem, in the short term. However, non-disclosure can have significant adverse psychological and public health consequences. Previous research has shown that HIV status disclosure is negatively correlated with self-stigma (Nobre et al., 2018). Non-disclosure to significant others and sexual partners may abrogate possible sources of social support which might normally buffer the negative psychological effects of stigma. Indeed, social support is proven to be protective against threats to self-esteem and psychological adversity (Crabtree et al., 2010). Moreover, on the level of public health, non-disclosure of one's HIV status to sexual partners could lead to onward HIV transmission if the person living with HIV has a detectable viral load. The benefits of HIV concealment motivation are limited and the adverse by-products of this self-protection strategy are significant. 


\section{Limitations}

This qualitative study has several limitations which should be addressed in future research. First, the sample included individuals who were diagnosed prior to the introduction of effective ART (pre-1996) and those diagnosed during the era of ART (post-1996). It is likely that the perception and experience of HIV stigma among those individuals who received their HIV diagnosis in the pre-ART era when HIV constituted a terminal condition differ from those diagnosed in the era of effective ART. A larger sample of participants living with HIV, stratified by time of diagnosis, would enable us to identify the effect of time of diagnosis on perception and experience of HIV. Second, all of the participants were engaging with HIV care and virally suppressed. In this article, it is noted that stigma leads to HIV concealment motivation and it is speculated on the basis of previous research (e.g. Jaspal, 2018) that, in some cases, stigma and non-disclosure could lead to disengagement from HIV care in this population. This hypothesis should be tested in individuals living with HIV in Finland who are not currently engaging with HIV care. Third, the small sample size in this qualitative study makes it impossible to generalize the results to the broader population. The use of quantitative methods to analyze data from a larger sample of gay men living with HIV in Finland would be a beneficial step towards generalizability beyond this participant sample.

\section{Conclusions}

Although Finland has a small and concentrated HIV epidemic, it has not yet achieved the UNAIDS 90-90-90 targets. HIV concealment motivation may impede the achievement of these targets. First, gay men living with HIV will be less able to access social support to cope with psychological stressors associated with both their sexual orientation and HIV status. This in turn may lead to decreased self-care behavior, disengagement from healthcare and engagement in risk-taking behavior as a coping strategy (Jaspal, 2018). Second, although all of the participants in this study had an undetectable viral load, it is possible that HIV concealment motivation could lead to onward HIV transmission in those who do not have an undetectable viral load. Individuals may make erroneous assumptions about their partner's HIV status and engage in risk behavior in the absence of open discussions about HIV and sexual health. Indeed, HIV stigma does preclude self-disclosure and open discussion. Consequently, it would be advantageous to raise further awareness and understanding of HIV in Finnish society, in which the HIV epidemic is small and not a public health priority. It is necessary to challenge HIV stigma. It is vital to facilitate access to social support among those who are diagnosed with HIV. This multi-pronged approach will undoubtedly reduce the risk of HIV concealment motivation among gay men living with HIV and, thus, lead to more favorable psychological and public health outcomes.

\section{Acknowledgements}

The author would like to thank Panda Eriksson who conducted and transcribed the interviews.

\section{References}

Crabtree, J. W., Haslam, A. S., Postmes, T., \& Haslam, C. (2010). Mental health support groups, stigma and self-esteem: Positive and negative implications of group identification. Journal of Social Issues, 66(3), 553-569. https://doi.org/10.1111/j.1540-4560.2010.01662.x

Daramilas, C., \& Jaspal, R. (2017). Measuring patient satisfaction: Insights from social psychology. Social Psychological Review, 19(1), 20-35. 
Davey, M. P., Foster, J., Milton, K., \& Duncan, T. M. (2009). Collaborative approaches to increasing family support for HIV positive youth. Families, Systems, \& Health, 27(1), 3952. https://doi.org/10.1037/a0014910

Dodge, R., Daly, A. P., Huyton, J., \& Sanders, L. D. (2012). The challenge of defining wellbeing. International Journal of Wellbeing, 2(3), 222-235.

https://doi.org/10.5502/ijw.v2i3.4

Duffy, L. (2005). Suffering, shame, and silence: the stigma of HIV/AIDS. Journal of the Association of Nurses in AIDS Care, 16(1), 13-20.

https://doi.org/10.1016/j.jana.2004.11.002

Earnshaw, V. A., Lang, S. M., Lippitt, M., Jin, H., \& Chaudoir, S. R. (2015). HIV stigma and physical health symptoms: Do social support, adaptive coping, and/or identity centrality act as resilience resources? AIDS and Behavior, 19, 41-49.

https://doi.org/10.1007/s10461-014-0758-3

Finnish Institute for Health and Welfare (2020). HIV and AIDS prevalence and statistics. https://thl.fi/fi/web/infektiotaudit-ja-rokotukset/taudit-ja-torjunta/taudit-ja-taudinaiheuttajata-o/hiv/hiv-ja-aids-esiintyvyys-ja-tilastot

Henry, K. L., Thornberry, T. P., \& Lee, R. D. (2015). The protective effects of intimate partner relationships on depressive symptomatology among adult parents maltreated as children. Journal of Adolescent Health, 57, 150-156.

https://doi.org/10.1016/j.jadohealth.2015.02.015

Hivpoint (2015). Rights of people living with HIV. https://hivpoint.fi/en/hiv-positive/rightspeople-living-hiv/

Holmberg, V., Soini, H., Kivelä, P., Ollgren, J., \& Ristola, M. (2019). Epidemiology and outcome of HIV patients in Finland co-infected with tuberculosis 1998-2015. BMC Infectious Diseases, 19, 264. https://doi.org/10.1186/s12879-019-3890-x

Jackson, S. D., \& Mohr, J. J. (2016). Conceptualizing the closet: Differentiating stigma concealment and nondisclosure processes. Psychology of Sexual Orientation and Gender Diversity, 3(1),80-92. https://doi.org/10.1037/sgd0000147

Jaspal, R. (2018). Enhancing Sexual Health, Self-Identity and Wellbeing among Men Who Have Sex With Men: A Guide for Practitioners. London: Jessica Kingsley Publishers.

Jaspal, R. (2020). Content analysis, thematic analysis and discourse analysis. In G. M. Breakwell, D. B. Wright \& J. Barnett (eds.), Research Methods in Psychology (5th Edition) (pp. 285-312). London: Sage.

Jaspal, R., \& Williamson, I. (2017). Identity management strategies among HIV-positive Colombian gay men in London. Culture, Health and Sexuality: An International Journal for 
Research, Intervention and Care, 19(2), 1374-88.

https://doi.org/10.1080/13691058.2017.1314012

Kluger, K., Blomqvist, C., \& Kivelä, P. (2019). Kaposi sarcoma in Southern Finland (20062018). International Journal of Dermatology, 58(11), 1258-1263.

https://doi.org/10.1111/ijd.14563

Korhonen, T., Kylmä, J., Houtsonen, J., Välimäki, M., \& Suominen, T. (2012). University students' knowledge of, and attitudes towards, HIV and AIDS, homosexuality and sexual risk behaviour: a questionnaire survey in two Finnish universities. Journal of Biosocial Science, 44(6), 661-675. https://doi.org/10.1017/S0021932012000338

Miller, A. K., Lee, B. L., \& Henderson, C. E. (2012). Death anxiety in persons with HIV/ AIDS: A systematic review and meta-analysis. Death Studies, 36(7), 640-663.

https://doi.org/10.1080/07481187.2011.604467

Nishimura, S., Nevgi, A. \& Tella, S. (2008). Communication style and cultural features in high/low context communication cultures: A case study of Finland, Japan and India.

Proceedings of a subject-didactic symposium in Helsinki, 2, 783-796.

https://people.uwec.edu/degravjr/GEOG-ANTH\%20351/Articles/NishimuraNevgiTella-

highcontextlowcontext.pdf

Nobre, N. R., Kylmä, J., Kirsi, T., \& Pereira, M. (2016). Social networks of older adults living with HIV in Finland. AIDS Care, 28(2), 186-190.

https://doi.org/10.1080/09540121.2015.1071774

Nobre, N., Pereira, M., Roine, R.P ., Sutinen, J., \& Sintonen, H. (2018). HIV-related selfstigma and health-related quality of life of people living with HIV in Finland. Journal of the Association of Nurses in AIDS Care, 29(2), 254-265.

https://doi.org/10.1016/j.jana.2017.08.006

Peterson, J. L. (2009). "You have to be positive." Social support processes of an online support group for men living with HIV. Communication Studies, 60(5), 526-541.

https://doi.org/10.1080/10510970903260368

Peyre, M., Gauchet, A., Bissuel, F., Blanc, M., Boibieux, A., Cotte, L., ... Epaulard, O. (2019). Satisfaction with sexual life in people living with HIV/AIDS: the persistent weight of the fear of transmission. AIDS Care, 31(6), 681-686.

https://doi.org/10.1080/09540121.2018.1537465

Rodger, A. J., Cambiano, V., Bruun, T., Vernazza, P., Collins, S., Degen, O., ... PARTNER Study Group (2019). Risk of HIV transmission through condomless sex in serodifferent gay couples with the HIV-positive partner taking suppressive antiretroviral therapy (PARTNER): final results of a multicentre, prospective, observational study. Lancet, 393(10189), 2428-2438. https://doi.org/10.1016/S0140-6736(19)30418-0 
Smit, P. J., Brady, M., Carter, M., Fernandes, R., Lamore, L., Meulbroek, M., ... Thompson, M. (2012) HIV-related stigma within communities of gay men: a literature review. AIDS Care, 24(4), 405-412. https://doi.org/10.1080/09540121.2011.613910

Suominen, T., Koponen, N., Mockiene, V., Raid, U., Istomina, N., Vänskä, M. L., BlekVehkaluoto, M., \& Välimäki, M. (2010). Nurses' knowledge and attitudes to HIV/AIDS-an international comparison between Finland, Estonia and Lithuania. International Journal of Nursing Practice, 16(2), 138-147.

https://doi.org/10.1111/j.1440-172X.2010.01822.x

Suominen, T., Heikkinen, T., Pakarinen, M., Sepponen, A. M., \& Kylmä, J. (2017).

Knowledge of HIV infection and other sexually transmitted diseases among men who have sex with men in Finland. BMC Infectious Diseases, 17(1), 121.

https://doi.org/10.1186/s12879-017-2203-5

Välimäki, M., Makkonen, P., Mockiene, V., Aro, I., Blek-Vehkaluoto, M., Istomina, N., Kisper-Hint, I. R., Staniuliene, V., Koponen, N., Vänskä, M. L., \& Suominen, T. (2010). Nursing and midwife students' willingness to provide care to patients with HIV/AIDS--a comparative study in Finland, Estonia and Lithuania. Nurse Education Today, 30(7), 674679. https://doi.org/10.1016/j.nedt.2010.01.004

Varni, S. E., Miller, C. T., McCuin, T., \& Solomon, S. (2012). Disengagement and engagement Coping with HIV/AIDS stigma and psychological well-being of people with HIV/AIDS. Journal of Social and Clinical Psychology, 31(2), 123-150. https://doi.org/10.1521/jscp.2012.31.2.123

Warner, L. R., \& Shields, S. A. (2013). The intersections of sexuality, gender, and race: Identity research at the crossroads. Sex Roles: A Journal of Research, 68(11-12), 803-810. https://doi.org/10.1007/s11199-013-0281-4

\footnotetext{
${ }^{1}$ There is evidence an individual who has had an undetectable HIV viral load for at least six months cannot transmit HIV to their sexual partners - this has been found to be the case in both heterosexual and gay male couples (Rodger et al., 2019).
} 